\title{
Essential validation of gene trap mouse ES cell lines: a test case with the gene Ttrap
}

\author{
LIESBETH VERMEIRE1,3, ABDELILAH IBRAHIMI1,3, THIERRY VOET 2,3, LIEVE UMANS ${ }^{1,3}$, \\ KATHLEEN CODDENS ${ }^{1,3}$, ANNICK FRANCIS ${ }^{1,3}$, TOM VAN DE PUTTE ${ }^{1,3}$, LEO A. VAN GRUNSVEN ${ }^{1,3}$, \\ AN ZWIJSEN ${ }^{1,3}$, DANNY HUYLEBROECK ${ }^{*}, 1,3$ and LUC NELLES ${ }^{1,3}$ \\ ${ }^{1}$ Laboratory of Molecular Biology (Celgen) and ${ }^{2}$ Human Genome Laboratory, Centre of Human Genetics, \\ KULeuven, ${ }^{3}$ Department of Molecular and Developmental Genetics (VIB11), VIB, Leuven, Belgium
}

\begin{abstract}
Gene trapping in mouse embryonic stem (ES) cells enables near-saturation vectorbased insertional mutagenesis across the genome of this model organism. About 135,000 trapped ES cell lines are made available to the scientific community by the International Gene Trap Consortium (IGTC; www.genetrap.org). A search of one of its databases identified an ES cell line (RRS512) with a $\beta$ Geo-based gene trap (gt) vector insertion in intron 5 of $T$ trap, a gene that encodes an intracellular signalling protein, which is implicated in gastrulation movement and left-right asymmetry in zebrafish embryos. We have determined the exact gt insertion point in the mutant ES cell clone RRS512 and confirmed the production of a chimaeric transcript consisting of the upstream Ttrap exons and the gene trap vector encoded marker/selection fusion sequences. This ES cell line was used to generate heterozygous Ttrap mutant mice, which were further crossed to obtain Ttrap ${ }^{g t / g t}$ mice. In contrast to Ttrap's documented essential role during nodal and Smad3 controlled zebrafish early embryogenesis, Ttrap $^{g t / g t}$ mice were born with a normal Mendelian distribution. However, subsequent analysis of these $T_{t r a p^{g t} / g t}$ mice has revealed a duplication of the wild-type Ttrap allele that was already present in the RRS512 cell line. Based on our detailed analysis presented here, we suggest an extensive procedure for the characterization of gene trap ES cell lines prior to generating gene trap mice with these.
\end{abstract}

KEY WORDS: duplication, EapII, ES cell, gene trap, Ttrap

\section{Introduction}

Since the near-completion and subsequent annotation of the human genome project (International Human Genome Sequencing Consortium, 2001, 2006; Venter et al., 2001), a large number of novel genes have been identified whose possible functions in development and disease remain largely unknown. Loss-offunction approaches in the mouse, including by mutagenesis of these genes, are relevant ways to obtain insight into the functions of such genes. Mutant mice can be generated via homologous recombination (gene targeting) or random mutagenesis (ENUbased mutagenesis, gene trapping) in ES cells (Skarnes, 2005). Gene targeting enables the deletion of either the entire gene or one or more critical exons of the gene, but also the introduction of subtle alterations such as a missense mutation. An appropriate targeting vector must be generated for each specific gene but can be constructed for any gene in the genome independent of the transcriptional status of the gene. Even though some of these vector construction steps are currently being fully automated and therefore becoming applicable at large scale, this approach remains labour-intensive. This is due to the need for screening a large number of ES cell colonies in order to select the correctly targeted ones, including in the case of conditional targeting, where the conditional status of the target allele has to be rigorously confirmed (e.g. the loxP site should flank at each side the

\footnotetext{
Abbreviations used in this paper: $\beta$ Geo, $\beta$-galactosidase neomycin-resistance fusion protein; EAPII, Ets-associated protein II; ES cell, embryonic stem cell; FISH, fluorescence in situ hybridization; Gapdh, glyceraldehyde-3phosphate-dehydrogenase; gt, gene trap; ISH, in situ hybridization; npt, neomycin phosphotransferase; SA, splice acceptor; Ttrap, TRAF and TNF receptor-associated protein; Zfhxla, Zinc finger homeobox gene la.
}

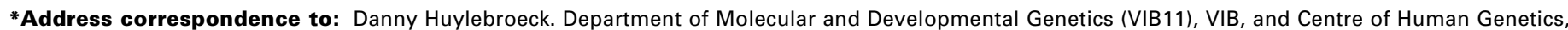
KULeuven, Campus Gasthuisberg, Bldg. Ond\&Nav1 PB812, Herestraat 49, B-3000 Leuven, Belgium. Tel: +32-16-345916. Fax: +32-16-345933.

e-mail: danny.huylebroeck@med.kuleuven.be
} 
critical exon and none should get lost during the recombination).

Gene trapping as a global method is conceptually better suited for high-throughput mutagenesis and can in fact come in many variants (i.e. enhancer trap, promoter trap, poly-A addition trap and gene trap, respectively). True gene trapping in ES cells relies on the disruption of functional transcription units by random integration of a promoter-less reporter construct without the need for detailed prior information on the structure of the gene (for review see Stanford et al., 2001). Many gene trap vectors contain a LacZ-neomycin phosphotransferase (npt) reporter sequence encoding for a $\beta$-galactosidase (from $E$. coll) -neomycin resistance ( $\beta \mathrm{Geo})$ fusion protein. The latter sequence is flanked at the 5 '-end by a splice acceptor (SA) site and at the 3'-end by a polyadenylation signal. Upon integration in an intron, the reporter gene is transcribed from the upstream endogenous promoter, yielding a transcript that fuses the upstream exons of the trapped gene with the reporter sequence, thereby simultaneously mutating the trapped gene and reporting its expression, including its pattern during embryogenesis if the corresponding gene trap ES cell line is used to generate heterozygous mice. Because gene trap vectors insert near-randomly across the genome, very large numbers of mostly individual gene trap mutations can be generated in ES cells through reasonably low numbers of experiments. This approach remains limited to genes that are transcribed in ES cells, because the insertion of SA site-containing gene trap vectors has to occur in an intron, and only expressed genes will be selected based on the expression of the dominant selectable marker npt. Another characteristic of such vectors is that alternative splicing may occur, leading to residual levels of wildtype mRNA and therefore the production of hypomorphic alleles (Voss et al., 1998a). This could be of potential interest to model certain diseases. Whereas homozygous Pkd1 knockout mice are embryonic lethal, homozygous hypomorphic Pkd1 mice are viable and show pathogenic features similar to the human autosomal dominant polycystic kidney disease (ADPKD; Lantinga-van Leeuwen et al., 2004). Hypomorphic alleles can also be used to circumvent early embryonic lethality caused by the targeted null allele. Whereas null mutants for noda/do not develop beyond gastrulation, hypomorphic mutants show evidence for nodal-mediated regulation of anterior-posterior axial positioning, anterior and midline patterning, and confirms a crucial role in mediating left-right patterning of the viscera (Lowe et al., 2001).

The major gene trapping laboratories have meanwhile joined in the International Gene Trap Consortium (Nord et al., 2006; www.genetrap.org), which now encompasses about 135,000 trapped ES cell lines that are made available to the biomedical research community.

TRAF and TNF receptor-associated protein (Ttrap) was identified as a novel CD40-binding protein (Pype et al., 2000) and as a partner for Ets transcription factors (EAPII; Pei et al., 2003). Recently, we showed that morpholino-mediated knockdown of this novel Smad-interacting protein in zebrafish leads to embryonic defects in convergent extension movement and left-right asymmetry axis formation due to aberrant nodal->Alk4->Smad3 signalling (Esguerra etal., 2007). Here, we characterize a Ttrap gene trap ES cell line obtained for the generation of homozy- gous Ttrapgt/gt mice.

\section{Experimental Protocols}

\section{Generation of Ttrap knockout mice, histology and in situ hybridization}

The ES cell line RRS512 (an E14 ES cell line from 129/ola mice; Baygenomics) was aggregated with CD1 acceptor morulae to generate chimaeric mice as described in Wood et al. (1993).

For in situhybridization (ISH), embryos were dissected in PBS and fixed overnight in $4 \%$ paraformaldehyde. Paraffin sections of embryos were subjected to ISH with an antisense riboprobe, representing the full-length $L a c Z$ or Ttrap gene, labelled with digoxigenin-UTP (Roche, Mannheim, Germany). This was done in the Ventana Discovery ${ }^{\mathrm{TM}}$ automated ISH instrument (Ventana Medical Systems, Tucson, AZ, USA). Noon of the day on which a copulation plug was observed, was termed embryonic day (E) 0.5.

\section{Genotyping}

Genomic DNA was isolated from ES cells, and from tail and yolk sac biopsies, using standard techniques. To distinguish between the mutant and wild-type alleles, a standard PCR strategy was designed using a common forward primer combined with reverse primers specific for each allele. The primer sequences were, respectively:

Forward (fwd):

5'-GAAGGCATGGCAGTGGAGGTGTGA-3';

Wild-type reverse (WT rev):

5'-CCACTACCTCTCCACACTCAGGATGGTG-3';

Mutant reverse (mut rev):

5'-TACTTTCGGTTCCGTCCTGGCTGC-3'.

These primers amplified a $570 \mathrm{bp}$-long fragment indicative for the wild-type Ttrap allele and a $350 \mathrm{bp}$-long for the mutant allele.

\section{RT-PCR analysis}

Total RNA was extracted from ES cells or adult mouse tissues using the RNeasy Mini Kit (Qiagen, Valencia, CA). One $\mu$ g of RNA was used as a template for cDNA synthesis using Superscript II reverse transcriptase (Invitrogen, Carlsbad, CA) and was primed with a mix of oligo-dT and random hexamers. Mouse glyceraldehyde-3-phosphate-dehydrogenase (Gapdh) was used as the reference gene. The primer sequences were in these cases:

Gapdh forward:

5'-AAGAAGGTGGTGAAGCAGGC-3';

Gapdh reverse:

5'-GCCTCTCTTGCTCAGTGTCC-3';

Ttrap exon 5 forward (R1):

5'-TTTCACAGCTATACTATTGAAGAAAGGAAGAGTG-3';

LacZreverse (R2):

5'-TTTGAGGGGACGACGACAGTATC-3';

Ttrap exon7 reverse (R3):

5'-CGATGCTTATAAGCAGCAGGGATCC-3'.

\section{Southern blotting}

Southern analysis was performed as described (Sambrook et al., 1989). Briefly, EcoRV-or Bcll-digested gDNA was separated by agarose gel electrophoresis and transferred to Nylon filters (Perkin-Elmer Life Sciences, Billerica, MA). Hybridization was done at $60^{\circ} \mathrm{C}$ with an $\alpha-\left[{ }^{32} \mathrm{P}\right]-\mathrm{dCTP}$ labelled probe either to the first 
800 bp of the $L a c Z$ gene or to intron 5 of Ttrap.

\section{Quantitative PCR analysis}

qPCR was performed on genomic DNA from tail biopsies on an ABI Prism 7000 (Applied Biosystems, USA) using SYBR Green PCR Master Mix (Invitrogen). The relative expression level of each gene was normalized to steady-state levels of Zinc finger homeobox gene 1a (Zfhx1a)mRNA. The primer sequences were as follows:

Zfh $x$ 1a forward: 5'-GGTTCACAGCCGTTTTCCAA-3';

Zfh $x$ 1a reverse: 5'-GTTCATCACCTGGGTCCGTAA-3';

Ttrap intron 5 forward (sg1):

5'-GGGTGGTGTGAGAAACAGTTATTTTA-3';

Gene trap intron reverse (sg2):

5'-TGTCTCCAAAGTTGATTCATGCTT-3';

Ttrap intron 5 reverse $(\mathrm{sg} 3)$ :

5'-TGTGCTGAATAAAGGAGACAGGAGAA-3';

Npt forward (sg4): 5'-TTGGCGGCGAATGGG-3';

Nptreverse (sg5): 5'-AATCGGGAGCGGCGAT-3'.

\section{Fluorescence in situ hybridization (FISH)}

FISH, microscopic evaluation and image recording was performed as described in Voet et al. (2003). The probe was labelled with biotin-16dUTP (Invitrogen) using the Nick Translation System (Invitrogen). Chromosomes were counterstained with DAPI.

\section{Statistical analysis}

The statistical analysis was performed by a two-tailed Student's ttest. Probability values of $p \leq 0.05$ were considered as significant.

\section{Results and Discussion}

\section{Molecular analysis of the gene trap locus}

Diagnostic and searchable gene trap sequences deposited in the Baygenomics gene trap database (Stryke et al., 2003; http:// baygenomics.ucsf.edu) are obtained through rapid amplification of 5 ' $\mathrm{CDNA}$ ends (5'RACE) PCR products obtained from RNA preparations of ES cell lines that contain insertions of the SABGeo-based trapping construct pGTOlxf. A search of this database for ES cell lines containing an insertion in the Ttrapgene yielded initially one entry, ES cell clone RRS512, which contains an insertion in Ttrap intron 5 ( Ttrap contains 7 exons; accession number NC_000079). Fig. 1A shows a schematic representation of this trapping event. To determine the exact genomic location of the insertion in this ES cell line, we screened intron 5 of Ttrap by PCR combining various forward primers in this intron with reverse primers encompassing the gene trap vector. Several such primer pairs generated PCR fragments, which were sequenced. The insertion of the vector occurred after bp 7823 in intron 5, and the $\mathrm{SA}$ sequence in front of the $\beta \mathrm{Geo}$-coding sequence was preserved (Fig. 1B). Furthermore, we confirmed by RT-PCR on ES cell RNA that the insertion resulted in a fusion transcript between sequences of exon 5 of Ttrapand the LacZ-nptreporter gene (Fig. $1 \mathrm{C})$, which was in agreement with the 5'RACE results from Baygenomics. We therefore used this ES cell line to generate a Ttrap mutant mouse.

\section{Generation and analysis of Ttrap gene trap mice}

RRS512 ES cells were aggregated with CD1 acceptor morulae

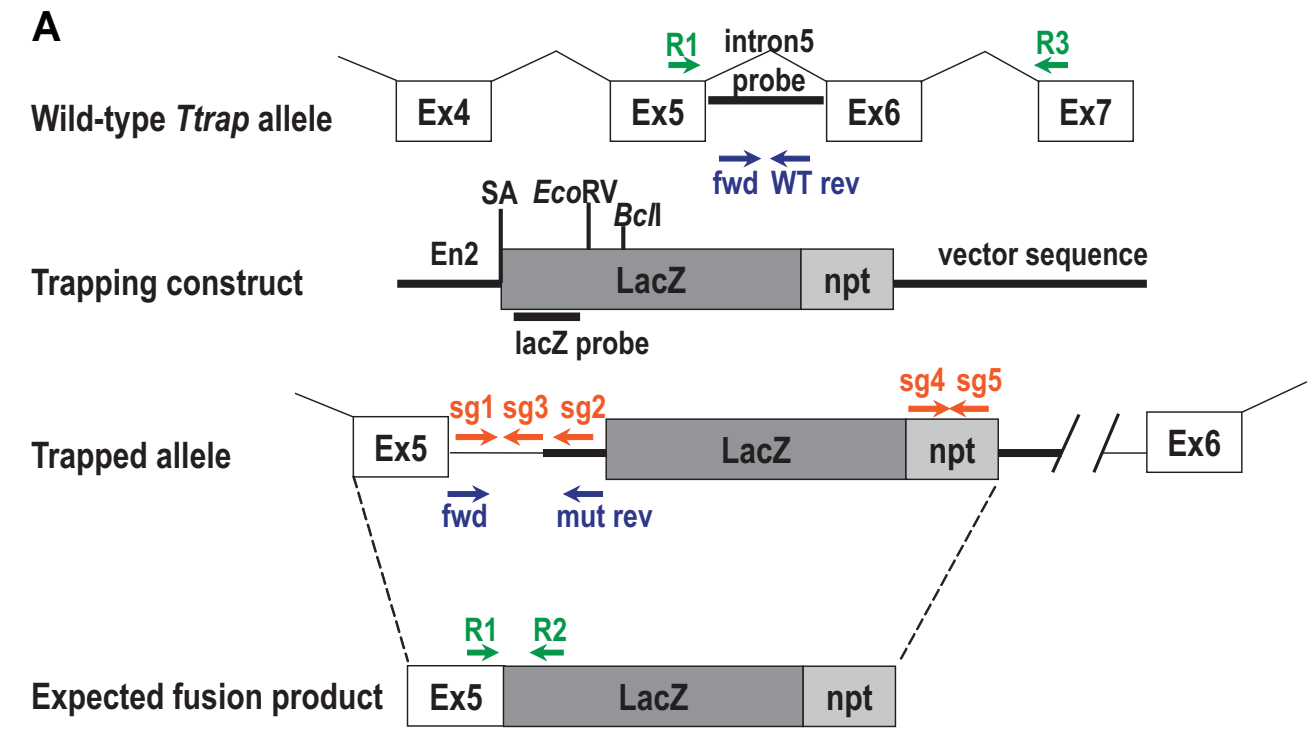

B aaaggaagcacccaggacaaaccttgcaagttgtagcaagggtaaacctgtgctgtggatgagcttatgttcagggtggtgtgagaaacagttatttagaagcatgaatcaacttggagacatgcgggcccttc catcaaggccccttogctagaagoggaagagg ctggagaagg cccgaccatcccccttcg cag/........../gtggataagtag ctag actccagcaaccagtaacctctg cccttctcctccatgacaaccag gtcccaggtocogaaaaccaaagaagaagaacgcagatcgcatogataacttogtatagcatacattatacgaagttatogcagatctggactctagaggatcocgtcgttttacaacgtogtgactgggaaaa
Fig. 1. Validation of the gene trap event in RRS512 ES cells. (A) Schematic representation of the trapping event. The pGTOIxf trapping construct contains the intron from the Engrailed-2 (En2) gene including its splice acceptor (SA) sequence upstream of the LacZ-npt fusion gene. The primers used for genotyping (fwd, WT rev, mut rev), RT-PCR (R1 to R3) and qPCR (sg1 to sg5), and the expected fusion product are indicated. (B) Sequence analysis on RRS512 ES cell DNA. Sequences in black represent Ttrap, while the blue sequences are gene trap vectorencoded sequences. The SA sequence is underlined. (C) RT-PCR on control (i.e. Ttrap ${ }^{+/+}$) and RRS512 ES cell RNA (Ttrap+/gt). The primer pair R1-R2 yielded a 450 bp-long fusion mRNA fragment. As a positive control, mouse Gapdh RNA was amplified. A negative control performed without reverse transcription (RT-) is shown.

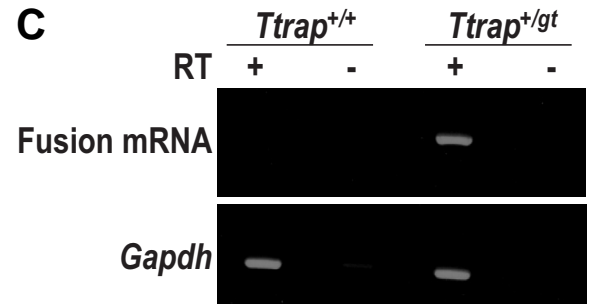


to generate chimaeric mice. Breeding of the chimaeras with CD1 females produced animals carrying the trapped allele, as was confirmed by PCR amplification of the npt sequence in tail DNA from 3-weeks old pups. Next, an allele-specific standard genotyping protocol was established to allow the discrimination between wild-type (Ttrap $\left.{ }^{+/+}\right)$, heterozygous (Ttrap $p^{+/ g t}$ ) and homozygous (Ttrap ${ }^{\text {tgt }}$ )animals. For this, we used a forward primer in intron 5, upstream of the insertion, in combination with a reverse primer either in intron 5 downstream of the insertion point (for the wildtype allele) or in the Engrailed-2 intron (which provides the SA) within the gene trap vector (for the trapped allele). These two PCR reactions were used to genotype the Ttrap gene trap mice (Fig. 2A). Of the 100 mice initially analyzed, every npt-positive mouse was always positive for the gene trap specific PCR product, suggesting a single gene trapping event in the RRS512 ES cells.

Gene trap vectors can be introduced into the ES cell genome by either electroporation or retroviral infection. The difference between the two strategies is the likelihood to obtain multiple integrations. While retroviral infection mostly leads to the integration of a single vector copy, electroporation of a plasmid-based vector can easily lead to multiple integrations in $20 \%$ of the trapped ES cells (Wiles et al., 2000; Stanford et al., 2001). Since the RRS512 ES cell line was generated by electroporation, we wanted to confirm a single insertion event. Therefore we performed Southern analysis with an internal LacZspecific probe on EcoRV-digested tail DNA (Fig. 2B) and ES cell DNA (data not shown). EcoRV recognizes only one site in the pGTOlxf gene trap vector (Fig. 1A). We obtained the expected $5.5 \mathrm{~kb}$-long band, but also an unexpected $7 \mathrm{~kb}$-long band, which suggests that at least two pGTOlxf vectors had integrated in the ES cell clone RRS512. To test the exact number of trapping vector copies, we performed quantitative (q)PCR analysis on genomic (g)DNA from Ttrap+lgt mice with a primer set specific for npt(Fig. 2C). Our data suggested that more than 40 copies of the vector had integrated. The presence of multiple integrations was confirmed by fluorescence in situhybridization (FISH) with vector pSA $\beta$ Geo as probe (Friedrich and Soriano, 1991). Clearly, the insertion occurred only in one chromosome (Fig. 2D), but the signal was too intense to represent only one copy, supporting the qPCR data. The resolution of FISH is too low for distinguishing between a tandem repeat and multiple insertion sites close to each other. The latter possibility seems unlikely, since after three backcrosses of Ttrap ${ }^{+/ g t}$ mice in CD1 background and analysis of 249 pups, there was no segregation of the insertion loci. Indeed, all nptpositive mice were still positive for the gene trap insertion in Ttrap and vice versa.

Because the $\beta$ Geo-encoding sequence is under the transcriptional control of the endogenous Ttrap promoter, we wanted to document the Ttrapgene expression domain during embryogenesis by staining for $\beta$-galactosidase. However, we failed to show any staining in the RRS512 ES cells and heterozygous embryos (data not shown), despite the fact that the RRS512 gene trap ES cells were selected based on their G418-resistance. Similar results have been reported in the literature (Voss et al., 1998b; Tsakiridis et al., 2007), making our results not entirely unexpected. Northern blot and in situhybridisation (ISH) data revealed however a widespread expression of TtrapmRNA during mouse and zebrafish embryogenesis (Pype et al., 2000; Esguerra et al., 2007). Since we detected a Ttrap- $\beta$ Geo fusion transcript at the RNA level in ES cells (Fig. 1C), we performed non-radioactive ISH on embryo sections with a $\beta$ Geo-specific (Fig. $3 \mathrm{~A}-\mathrm{C}$ ) and Ttrapspecific (Fig. 3D) antisense RNA probe. This confirmed the

A

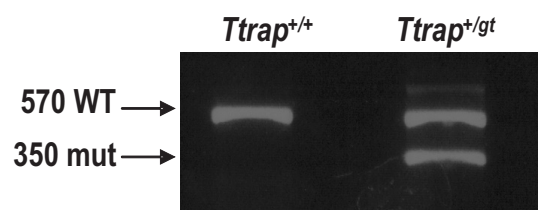

C

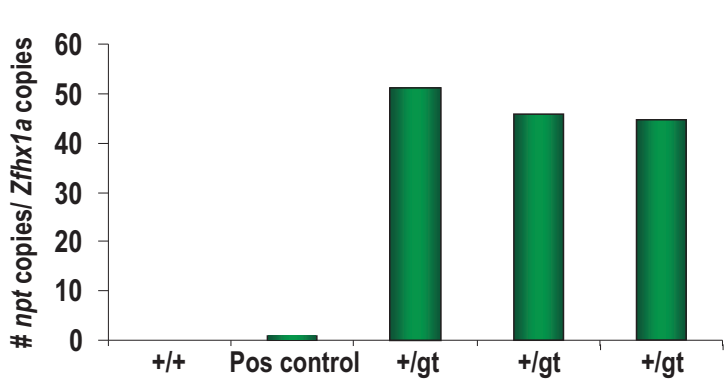

D

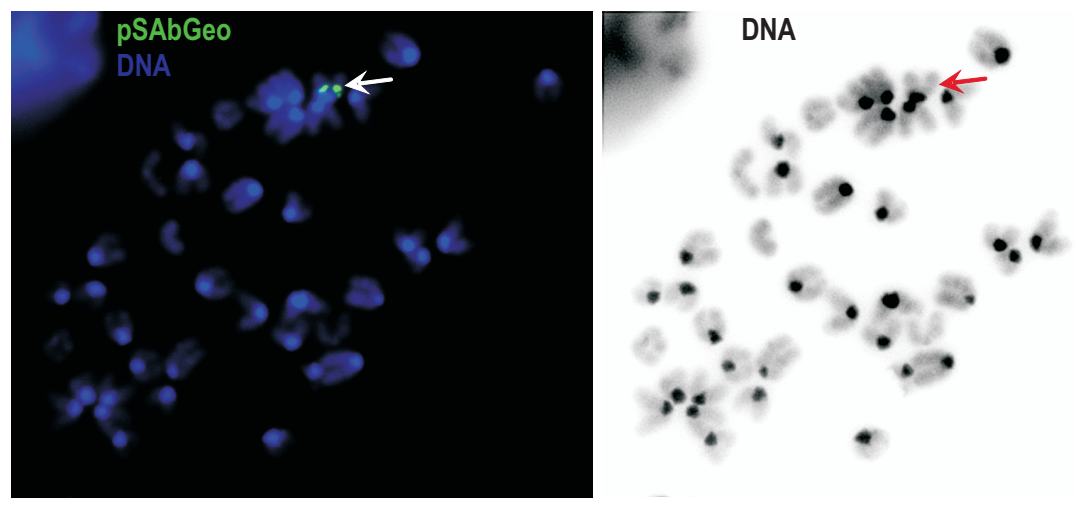

Fig. 2. Determination of the number of gene trap vector insertions. (A) PCR genotyping on offspring from heterozygous matings. The primer pairs fwd-WT rev and fwd-mut rev were mixed in a single PCR reaction to amplify a $570 \mathrm{bp}$-long WT and a $350 \mathrm{bp}$-long mutant specific fragment from gDNA. (B) Southern blot analysis on EcoRV-digested gDNA from wild-type (+/+) and heterozygous (+/gt) littermates using a LacZ DNA probe. (C) Quantitative PCR with primers specific for the npt gene (sg4-sg5) on gDNA from wild-type and heterozygous littermates, compared with a positive (pos) control sample having only 1 npt gene. Values are normalized to Zfhx1a. (D) Fluorescence in situ hybridization on metaphase chromosome spreads of RRS512 ES cells using biotin-labelled pSAbGeo (green) as probe (left panel). The chromosomes were counterstained with DAPI. The white arrow indicates the positive signal. Right panel: G-like banding derived from DAPI channel. The red arrow indicates the corresponding chromosome. 


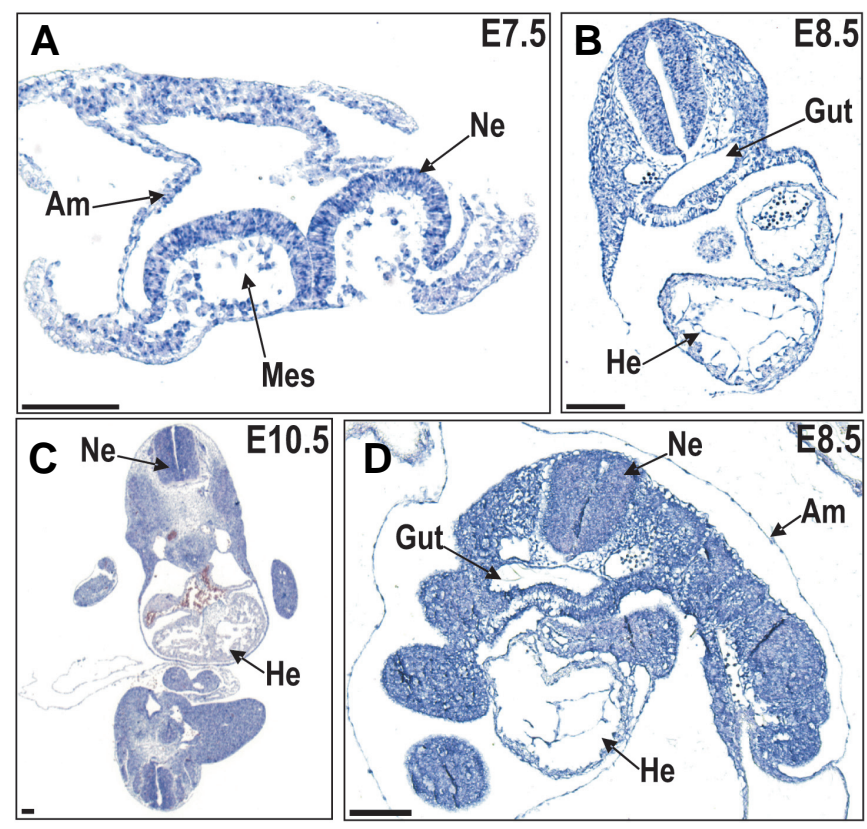

Fig. 3. Non-radioactive in situ hybridization, using a $\operatorname{LacZ}(\mathrm{A}-\mathrm{C})$ or a Ttrap (D) antisense probe, on Ttrap $^{+/ g t}$ embryos revealed ubiquitous expression of Ttrap transcript throughout the entire embryo. Blue is positive signal, pink is counterstaining with nuclear fast red. Scale bare represents $100 \mathrm{~mm}$. Am, amnion; He, heart; Mes, mesoderm; Ne, neurectoderm.

previously observed ubiquitous expression of TtrapmRNA throughout gestation.

\section{Ttrap ${ }^{\mathrm{gt} / \mathrm{gt}}$ mice derived from the RRS512 ES cell line are viable}

Ttrap ${ }^{+/ g t}$ mice were crossed to generate Ttrap ${ }^{g t g t}$ mutant mice. We did not obtain any homozygous gt/gt offspring at weaning $(n=118)$, while about 30 of these mutant animals were expected (Table 1). Backcrossing experiments showed that both females and males transmitted the gtallele (data not shown), suggesting that total Ttrap deficiency resulting from the gene trap vector insertion is embryonic lethal. To determine the stage of embryonic lethality, we collected embryos from heterozygous matings at different developmental stages (Table 1). Surprisingly, no Ttrapgtgt embryos were found by the standard PCR protocol, not even at the 2-cell stage. Up to mid 2-cell stage, embryos rely largely on maternal mRNA and proteins synthesized during oogenesis. Then, embryonic genes are switched on and most of the maternal mRNA is rapidly degraded. Maternal protein however can persist beyond this time (for review, see Schultz, 2002). Therefore, it is unlikely that our homozygous Ttrapgene trap embryos die earlier than the 2-cell stage.

Puzzled by this, we introduced an alternative genotyping protocol, i.e. GPCR analysis on gDNA, to determine the exact number of gene trap alleles (Fig. 4A, upper panel), as well as the corresponding number of nptcopies (Fig. 4A, lower panel) in gene trap littermates. This demonstrated that Ttrapgtgt offspring were actually born at the normal Mendelian distribution (Table 2). This viability was however in sharp contrast to the severe and multiple defects observed in Ttrapmorphant zebrafish embryos (Esguerra et al., 2007). Hence, we investigated whether Ttrapgtgt mice were completely devoid of wild-type Ttrap mRNA. Despite the many gene trap copies inserted in the Ttraplocus, RT-PCR analysis on Ttrap ${ }^{+/ t}$, Ttrap ${ }^{+/ g t}$ and Ttrapgtgt littermates showed irrefutably the presence of wild-type TtrapmRNA in the Ttrapgtgt mice (Fig. 4B). The Ttrap- $\beta$ Geofusion mRNA transcript could be detected in the heterozygous and homozygous mice, but not in the wild-type littermates, as expected. These observations were confirmed by Northern blot and RT-qPCR (data not shown).

There are multiple possible explanations for these results. Either a problem with the splicing occurred leading to the formation of wild-type mRNA, which is a problem that has been reported earlier (Voss et al., 1998b), or Ttrapgtgt mice were not real homozygous gene trap mice for Ttrap. To discriminate between these two possibilities, we performed Southern analysis on Bclldigested Ttrap ${ }^{+/}$, Ttrap ${ }^{+/ g t}$ and Ttrapgtgt gDNA with a Ttrapintron 5 specific probe that overlaps the insertion point. As shown in Fig. 4C, the $9.8 \mathrm{~kb}$-long wild-type band is clearly present in the $\mathrm{gt} / \mathrm{gt}$ mice, confirming the initial standard PCR results. This is then indicative for a duplication of the wild-type allele. We investigated this hypothesis using qPCR on gDNA of the different genotypes, using a primer pair located in intron 5 of Ttrapand which overlaps the gene trap insertion point. This analysis revealed equal amounts of wild-type alleles in Ttrapgtgt mice as compared with Ttrap ${ }^{+/ t}$ mice. When using a primer pair in intron 5 upstream of the gene trap insertion point, the double amount of intron 5 copies could be observed in Ttrapgtgt mice as compared with Ttrap ${ }^{+/}$mice (Fig. $4 \mathrm{D} ; \mathrm{p}=0.0000002)$. This is in contradiction with the expected equal amounts when no duplication is present. This was also the case for primer pairs in exon $1(p=0.000001)$, exon $2(p=0.0013)$ and exon $3(p=0.00014)$, but not for exon $7(p=0.53$; data not shown), which is indicative for only a partial duplication of the Ttrap allele. Since there is a fusion between exon 5 and exons $6 /$ 7 of Ttrap at the mRNA level in Ttrapgtgt mice (Fig. 4B, upper panel), we hypothesize that the duplication has occurred inbetween the gene trap vector insertion point and exon 6/7, leading to the formation of one intact wild-type allele on the gene trap

TABLE 1

\section{GENOTYPE DISTRIBUTION OF PUPS/EMBRYOS FROM HETEROZYGOUS MATINGS AT DIFFERENT STAGES, DETERMINED BY STANDARD PCR}

\begin{tabular}{lcccc} 
Stage & $+/+$ & $\mathbf{+ / g t}$ & $\mathbf{g t} / \mathbf{g t}$ & Total \\
\hline Weaning & 26 & 92 & 0 & 118 \\
\hline E12.5 & 27 & 64 & 0 & 91 \\
E10.5 & 32 & 64 & 0 & 96 \\
E8.5 & 12 & 48 & 0 & 60 \\
\hline E3.5 & 9 & 37 & 0 & 46 \\
E2.5 & 7 & 53 & 0 & 60 \\
2/4-cell & 17 & 51 & 0 & 68 \\
\hline
\end{tabular}

TABLE 2

\section{GENOTYPE DISTRIBUTION OF 3-WEEK OLD PUPS FROM HETEROZYGOUS MATINGS, DETERMINED WITH QUANTITATIVE PCR ON TAIL DNA}

\begin{tabular}{cccc} 
Genotype & No. & Expected (\%) & Actual (\%) \\
\hline +/+ & 24 & 25 & 20 \\
+/gt & 68 & 50 & 55 \\
gt/gt & 31 & 25 & 25 \\
\hline
\end{tabular}


A


C

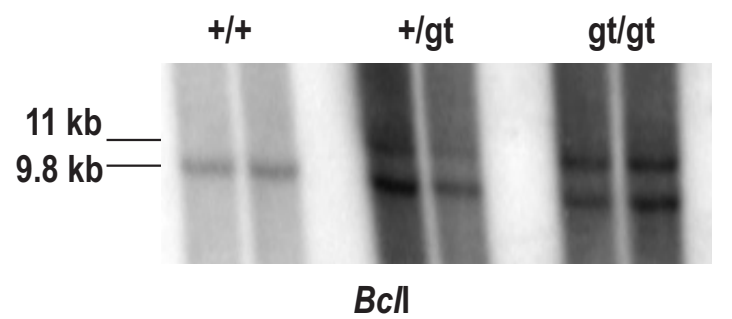

B
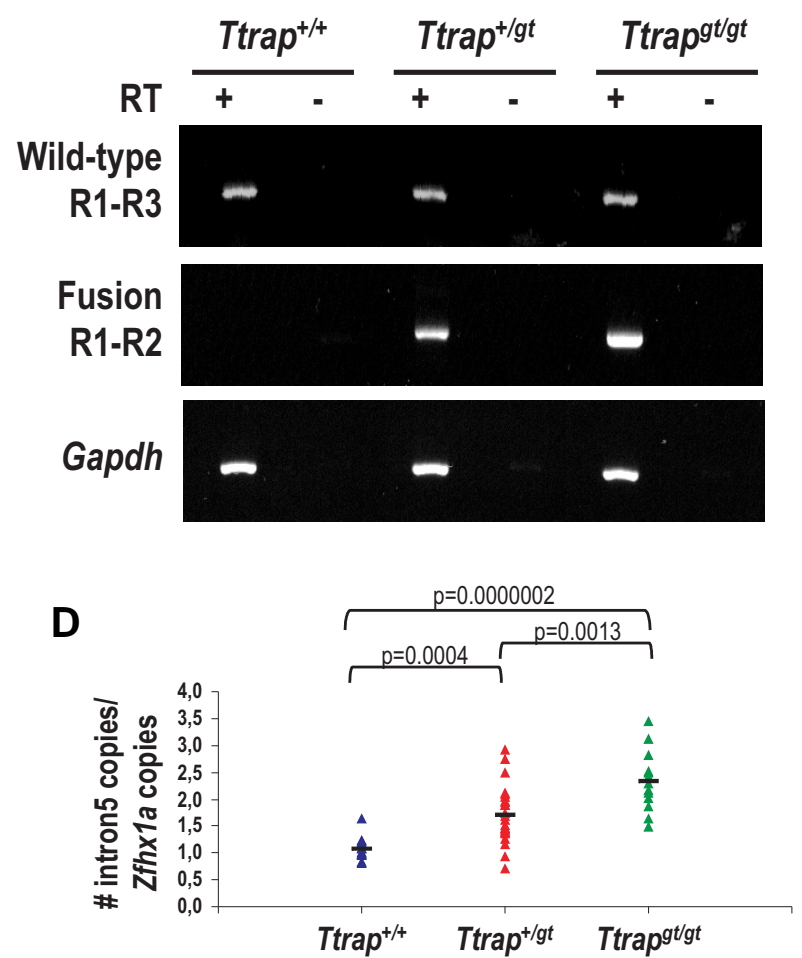

E

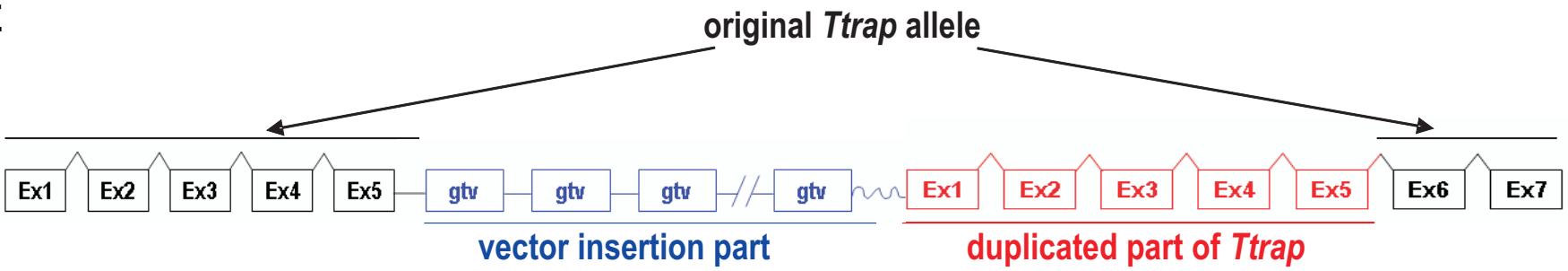

Fig. 4. Analysis of Ttrap $^{\mathbf{g t} / \mathbf{g t}}$ mice. (A) Quantitative PCR on tail gDNA from wild-type (+/+), heterozygous (+/gt) and homozygous (gt/gt) Ttrap littermates to determine the number of gene trap alleles (primers sg1-sg2; upper panel) and corresponding number of npt copies (primers sg4-sg5; lower panel). Values are normalized to Zfhx1a. (B) RT-PCR on adult kidney RNA from Ttrap ${ }^{+/}$, Ttrap ${ }^{+/ g t}$ and Ttrapgt/gt littermates. A 408 and a 450 bplong fragment represent wild-type Ttrap RNA and Ttrap-bGeo fusion RNA, respectively. The primers used are indicated. Mouse Gapdh RNA was used as positive control. (C) Southern blot analysis on Bcll-digested gDNA from wild-type (+/+), heterozygous (+/gt) and homozygous (gt/gt) littermates using a probe derived from Ttrap intron 5 . The $9.8 \mathrm{~kb}$ and $11 \mathrm{~kb}$ bands represent the wild-type and the gene trap allele, respectively. (D) Quantitative PCR on tail gDNA from wild-type ( $+/+; n=12)$, heterozygous ( $/$ /gt; $n=25)$ and homozygous (gt/gt; $n=13$ ) littermates with a Ttrap intron5 primer pair (sg1-sg3) upstream of the gene trap insertion point. Values are normalized to Zfhx1a. - represents the mean value. (E) Schematic representation of the gene trap allele with the hypothesized duplication. Gtv, gene trap vector.

allele (see schematic representation in Fig. 4E). These results clearly explain why wild-type Ttrap mRNA was detected in the Ttrapgtgt mice and why no embryonic lethal phenotype was obtained.

Gene trapping is a powerful large-scale mutagenesis approach that, in a single experimental set-up, can provide data on gene function and expression. Therefore it has been widely used both by large consortia of laboratories and by companies but also in focused screens of smaller research groups to generate gene trap ES cell lines and new mutant mouse models. Nevertheless, only about a year later, when homozygous mice are eventually available, one will know for sure whether the trapping of the gene leads to phenotypes arising from true loss-of-function of the gene. From the many thousands of gene trap ES cell lines that have been generated so far, only a small percentage has been successfully used to generate a knockout mouse (Mouse genome database; Forrai and Robb, 2005; Bult et al., 2008). Hence, it cannot be excluded that some of the available gene trap ES cell clones will not lead to a complete knockout mouse, including because of duplication events such as the one found here.

The standard operating procedure to characterize a gene trap ES cell line is considered as quite straightforward (Fig. 1), i.e 
carefully analyzing (i) the site of vector integration, (ii) the presence of the splice acceptor sequence and (iii) the synthesis of the expected fusion product. As our data with the RRS512 line clearly show, this procedure is insufficient: one should consider taking the analysis one step further and also check for (iv) duplications of the wild-type allele (by qPCR on gDNA), already at the ES cell level. When all these experiments give the expected results, one can confidently start with the generation of the gene trap mouse. Altogether, we conclude also that a gene trap ES cell line is not always the fastest way to generate a mutant mouse. Indeed, novel cloning techniques and homologous recombination in bacteria have now facilitated the design and construction of tailored gene replacement vectors, and this could potentially favour gene targeting over gene trapping for individual knockout projects. If gene targeting is however not an option, one should very thoroughly characterize the gene trap ES cell line to exclude the possibility of gene duplication that could hamper the generation of a loss-offunction mouse.

\section{Acknowledgments}

The authors would like to thank D. Célis, M. Missoul, A. Stryjewska and E. Maas formouse care; F. Cornelis for help with Southern blotting; L. Cox for help with qPCR analysis; and $R$. Chernomorsky and $A$. Economides for independently confirming the number of integration events. This work was supported by the EC FPG Integrated Project EndoTrack (LSHG-CT2006-019050).

\section{References}

BULT, C.J., EPPIG, J.T., KADIN, J.A., RICHARDSON, J.E., BLAKE, J.A. and THE MOUSE GENOME DATABASE, G. (2008). The Mouse Genome Database (MGD): mouse biology and model systems. Nucl. Acids Res. 36: D724-728.

ESGUERRA, C.V., NELLES, L., VERMEIRE, L., IBRAHIMI, A., CRAWFORD, A.D., DERUA, R., JANSSENS, E., WAELKENS, E., CARMELIET, P., COLLEN, D. et al. (2007). Ttrap is an essential modulator of Smad3-dependent Nodal signaling during zebrafish gastrulation and left-right axis determination. Development 134: 4381-4393.

FORRAI, A. and ROBB, L. (2005). The gene trap resource: A treasure trove for hemopoiesis research. Experimental Hematology 33: 845-856.

FRIEDRICH, G. and SORIANO, P. (1991). Promoter traps in embryonic stem cells: a genetic screen to identify and mutate developmental genes in mice. Genes Dev. 5: 1513-1523.

LANTINGA-VAN LEEUWEN, I.S., DAUWERSE, J.G., BAELDE, H.J., LEONHARD, W.N., VAN DE WAL, A., WARD, C.J., VERBEEK, S., DERUITER, M.C., BREUNING, M.H., DE HEER, E. et al. (2004). Lowering of Pkd1 expression is sufficient to cause polycystic kidney disease. Hum. Mol. Genet. 13: 3069-3077.

LOWE, L., YAMADA, S. and KUEHN, M. (2001). Genetic dissection of nodal function in patterning the mouse embryo. Development 128: 1831-1843.

NORD, A.S., CHANG, P.J., CONKLIN, B.R., COX, A.V., HARPER, C.A., HICKS G.G., HUANG, C.C., JOHNS, S.J., KAWAMOTO, M., LIU, S. et al. (2006). The International Gene Trap Consortium Website: a portal to all publicly available gene trap cell lines in mouse. Nucl. Acids Res. 34: D642-648.

PEI, H., YORDY, J.S., LENG, Q., ZHAO, Q., WATSON, D.K. and LI, R. (2003). EAPII interacts with ETS1 and modulates its transcriptional function. Oncogene 22: 2699-709.

PYPE, S., DECLERCQ, W., IBRAHIMI, A., MICHIELS, C., VAN RIETSCHOTEN, J.G., DEWULF, N., DE BOER, M., VANDENABEELE, P., HUYLEBROECK, D. and REMACLE, J.E. (2000). TTRAP, a novel protein that associates with CD40, tumor necrosis factor (TNF) receptor-75 and TNF receptor-associated factors (TRAFs), and that inhibits nuclear factor-kappa B activation. J Biol Chem 275 18586-93.

SAMBROOK, J., FRITSCH, E. and MANIATIS, T. (1989). Molecular Cloning: a Laboratory Manual Second Edition. New York, Cold Spring Harbor Laboratory Press Book 2: Section 9.38-9.40.

SCHULTZ, R.M. (2002). The molecular foundations of the maternal to zygotic transition in the preimplantation embryo. Hum Reprod Update 8: 323-331.

SKARNES, W.C. (2005). Two ways to trap a gene in mice. Proceedings of the National Academy of Sciences 102: 13001-13002.

STANFORD, W.L., COHN, J.B. and CORDES, S.P. (2001). Gene-trap mutagenesis: past, present and beyond. Nat Rev Genet 2: 756-768.

STRYKE, D., KAWAMOTO, M., HUANG, C.C., JOHNS, S.J., KING, L.A., HARPER, C.A., MENG, E.C., LEE, R.E., YEE, A., L'ITALIEN, L. etal. (2003). BayGenomics: a resource of insertional mutations in mouse embryonic stem cells. Nucl. Acids Res. 31: 278-281.

TSAKIRIDIS, A., TZOUANACOU, E., LARRALDE, O., WATTS, T.M., WILSON, V., FORRESTER, L. and BRICKMAN, J.M. (2007). A novel triple fusion reporter system for use in gene trap mutagenesis. Genesis 45: 353-360.

VENTER, J.C.ADAMS, M.D.MYERS, E.W.LI, P.W.MURAL, R.J.SUTTON, G.G.SMITH, H.O.YANDELL, M.EVANS, C.A.HOLT, R.A. et al. (2001). The Sequence of the Human Genome. Science 291: 1304-1351.

VOET, T., LIEBE, B., LABAERE, C., MARYNEN, P. and SCHERTHAN, H. (2003). Telomere-independent homologue pairing and checkpoint escape of accessory ring chromosomes in male mouse meiosis. Journal of Cell Biology 162: 795-808.

VOSS, A., THOMAS, T. and GRUSS, P. (1998a). Efficiency assessment of the gene trap approach. Developmental Dynamics 212: 171-180.

VOSS, A.K., THOMAS, T. and GRUSS, P. (1998b). Compensation for a gene trap mutation in the murine microtubule-associated protein 4 locus by alternative polyadenylation and alternative splicing. Developmental Dynamics 212: 258-266.

WILES, M.V., VAUTI, F., OTTE, J., FUCHTBAUER, E.-M., RUIZ, P., FUCHTBAUER A., ARNOLD, H.-H., LEHRACH, H., METZ, T., VON MELCHNER, H. et al. (2000). Establishment of a gene-trap sequence tag library to generate mutant mice from embryonic stem cells. Nat Genet 24: 13-14.

WOOD, S.A., ALLEN, N.D., ROSSANT, J., AUERBACH, A. and NAGY, A. (1993). Non-injection methods for the production of embryonic stem cell-embryo chimaeras. Nature 365: 87-89. 


\section{Further Related Reading, published previously in the Int. J. Dev. Biol.}

See our Special Issue Pattern Formation edited by Cheng-Ming Chuong and Michael K. Richardson at:

http://www.ijdb.ehu.es/web/contents.php?vol=53\&issue=5-6

See our Special Issue Stem Cells \& Transgenesis edited by Robert E. Hammer and Richard R. Behringer at:

http://www.ijdb.ehu.es/web/contents.php?vol=42\&issue=7

Generation and functional characterization of mice with a conditional BMP7 allele

Vasiliki Zouvelou, Ourania Passa, Katerina Segklia, Sotiris Tsalavos, David M. Valenzuela, Aris N. Economides and Daniel Graf Int. J. Dev. Biol. (2009) 53: 597-603

\section{Mouse induced pluripotent stem cells}

Eamon Geoghegan and Lucy Byrnes

Int. J. Dev. Biol. (2008) 52: 1015-1022

Inadvertent presence of pluripotent cells in monolayers derived from differentiated embryoid bodies

Miguel A. Ramírez, Eva Pericuesta, Raúl Fernández-González, Belén Pintado and Alfonso Gutiérrez-Adán

Int. J. Dev. Biol. (2007) 51: 397-408

Mouse models to study inner ear development and hereditary hearing loss Lilach M. Friedman, Amiel A. Dror and Karen B. Avraham Int. J. Dev. Biol. (2007) 51: 609-631

\section{For all the latest on Pattern Formation research, see our latest Special Issue edited by C.-M. Chuong and M.K. Richardson.}

http://www.ijdb.ehu.es/web/contents.php?vol=53\&issue=5-6



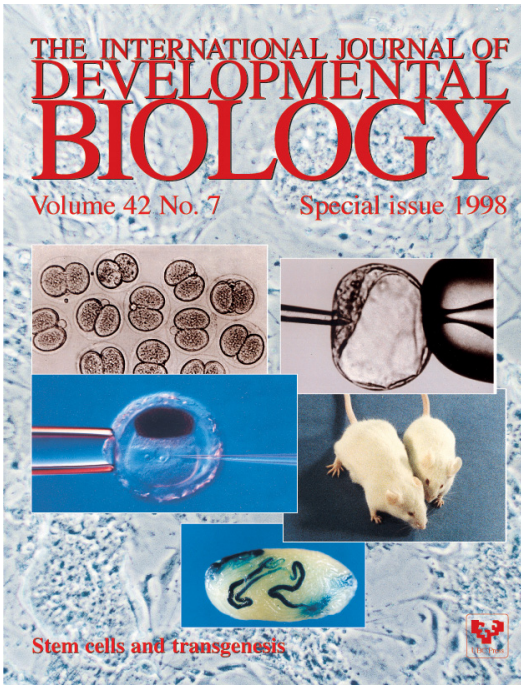

5 yr ISI Impact Factor $(2008)=3.271$

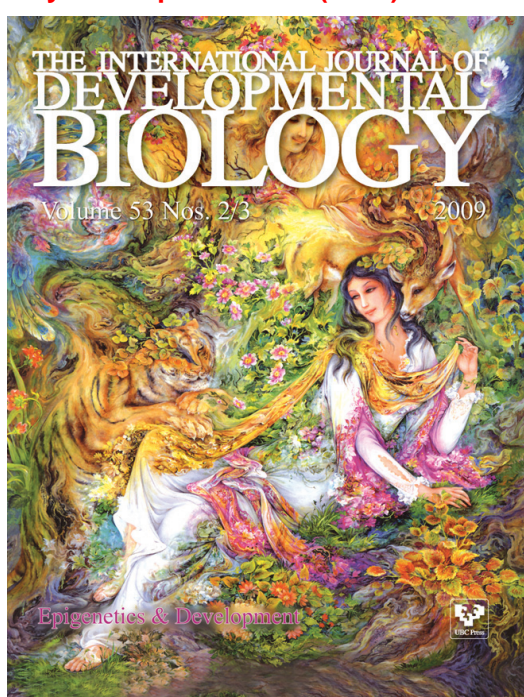

